\title{
Nucleon-Antinucleon Interaction from the Skyrme Model: II. Beyond the Product Ansatz
}

\author{
Yang Lu, Pavlos Protopapas and R. D. Amado \\ Department of Physics and Astronomy \\ University of Pennsylvania, Philadelphia, Pennsylvania 19104
}

September 13, 1997

\begin{abstract}
We calculate the full static interaction of a Skyrmion and an anti-Skyrmion as a function of separation and relative grooming. From this, using projection methods and Born-Oppenheimer mixing, we obtain the nucleon antinucleon interaction. We find agreement with the major features of the empirical interaction including the strong central attraction and the sharp onset of annihilation at about $1 \mathrm{fm}$.
\end{abstract}

\section{Introduction}

Making a theory of low energy nucleon-antinucleon annihilation from the entrance channel through to the annihilation products presents a daunting challenge. An exact QCD based calculation of the process is beyond our present analytical understanding and our computational resources. We are left then with either abandoning the problem or using some approximate or effective theory. Such a theory must include an account of the nature of the nucleon and the antinucleon, in order to describe annihilation, as well as containing a description of their interaction. The theory should also have its roots in QCD. The only such effective theory of annihilation that contains the requisite ingredients and gives hope of being tractable is based on the Skyrme model [1]. This models QCD in the classical or large number of colors $\left(N_{C}\right)$ limit and at long distances or low momentum [2, 3]. Sommermann et al. [4 have shown, in a detailed numerical investigation, that a Skyrme treatment of annihilation gives great insight into the process, and we have exploited that insight to show that the Skyrme treatment can account for the major features of the annihilation channels [5.

Thus far most of our results have come from a very simple picture of the the annihilation final state. We start with a spherical "blob" of Skyrmionic matter with zero baryon number and energy of two nucleon masses. We propagate this blob using the classical Skyrme equations, including classical vector meson fields, until it is well into the radiation zone and then use coherent states to reintroduce the meson quanta of the final state. This gives a remarkably good overall picture of the observed branching ratios seen in low energy nucleon antinucleon annihilation. However it does not give any insight into the incoming nucleon antinucleon channel. For this we have to study the initial state dynamics. A full dynamical Skyrmion calculation of the initial state is difficult and fraught with instabilities , 6 , 6. Therefore we take a simpler approach of using the Skyrme dynamics to obtain the nucleon antinucleon interaction potential. From that we later can connect the entrance channel to the annihilation blob and from there proceed as before.

Our previous attempt to obtain the nucleon antinucleon interaction from the Skyrme model was based on the product ansatz [7]. This calculation reproduces some of the major features of the observed interaction, 
but fails to give the observed strong central attraction. Such a failure is also present in the product ansatz approach to the nucleon nucleon interaction, [8], and is a failing of the ansatz, not of the Skyrme approach. In the nucleon nucleon case the full central attraction was obtained by taking two steps. First a full dynamical calculation of the interaction energy of two Skyrmions as a function of separation and relative grooming was done [9]. This is a difficult and numerically intensive calculation. Then the results of that calculation were combined with calculation of dynamical $\Delta$ mixing using the Born-Oppenheimer method, [10], to yield a nucleon nucleon interaction with most of the empirical features, in particular the strong central attraction. In this paper we report a calculation of the nucleon antinucleon interaction taking the corresponding two steps. First we compute the Skyrmion anti-Skyrmion interaction energy as a function of separation and relative grooming using the full Skyrme dynamics. As in the Skyrmion Skyrmion case, this is a difficult and complex computation. We then use Born-Oppenheimer techniques as well as standard Skyrmion to baryon projection methods to obtain the nucleon antinucleon potentials. We find strong central attraction between the nucleon and antinucleon of the form and magnitude seen from the data, as well as qualitative agreement with the other principal features of the empirical potentials. We also find strong and sudden coupling of the nucleons to the annihilation channel at about $1 \mathrm{fm}$, just as is seen in empirical fits to the data.

This paper is organized as follows. In the next section we present the major features of the formulation we use for the dynamical calculation. We do not go into great detail here since much of the formulation is already well presented in the literature. The following section reports the numerical methods we used. Since the calculation is challenging and difficult, we give some detail here. However the reader interested only in the finished answers can skip this section. We follow with a section on results that reports both our Skyrmion anti-Skyrmion interaction and our nucleon antinucleon interaction as well as showing some plots of Skyrme fields. We end with a discussion section that reviews briefly what our next steps might be.

\section{Formulation}

The Skyrme model, [1], is a classical, nonlinear field theory of an $\mathrm{SU}(2)$ valued field. The Lagrangian for the theory is written

$$
\mathcal{L}=-\frac{f_{\pi}^{2}}{4} \operatorname{Tr}\left[L_{\mu} L^{\mu}\right]+\frac{1}{32 e^{2}} \operatorname{Tr}\left[L_{\mu}, L_{\nu}\right]^{2}+\frac{m_{\pi}^{2} f_{\pi}^{2}}{2} \operatorname{Tr}[U-1]
$$

where $L_{\mu}$ is expressed in terms of the unitary $\mathrm{SU}(2)$ valued matrix $U$ by

$$
L_{\mu}=U^{+} \partial_{\mu} U
$$

The first term of the Lagrangian comes from the nonlinear sigma model. The second, the Skyrme term, was introduced by Skyrme to stabilize the model, and the third term is the pion mass term. It is the term that breaks chiral invariance. The three constants in the theory are the pion decay constant, $f_{\pi}$, the constant $e$ (not the electric charge) introduced by Skyrme to set the scale of his stabilizing term, and the pion mass, $m_{\pi}$. The Lagrangian admits topologically stable objects carrying a conserved winding number that Skyrme associated with baryon number. It is customary to adjust $f_{\pi}$ and $e$ to yield the correct long distance tail of the pion wave in the nucleon. We follow [9] in this and take $f_{\pi}=93 \mathrm{MeV}$ and $e=4.76$. We take the pion mass at its observed value?. The unitary $\mathrm{SU}(2)$ valued field $U$ can be written in terms of the chiral angle iso-vector field $\boldsymbol{F}$ as follows,

$$
U=\exp i \boldsymbol{\tau} \cdot \boldsymbol{F},
$$

\footnotetext{
${ }^{1}$ Note how few parameters are in the Skyrme model and note further that we adjust none of them to calculate the interaction energy.
} 
and then $L_{\mu}$ expressed in terms of $\mathbf{F}$ by

$$
L_{\mu}=i \boldsymbol{\tau} \cdot \boldsymbol{A}_{\mu}
$$

with

$$
\boldsymbol{A}_{\mu}=\partial_{\mu} \boldsymbol{F} \frac{\sin F \cos F}{F}+\boldsymbol{F} \partial_{\mu} F \frac{F-\sin F \cos F}{F^{2}}+\boldsymbol{F} \times \partial_{\mu} \boldsymbol{F} \frac{\sin ^{2} F}{F^{2}} .
$$

With these definitions, the terms in the Skyrme Lagrangian become, for the nonlinear sigma term

$$
\begin{aligned}
\mathcal{L}_{\sigma} & =\frac{f_{\pi}^{2}}{2} \boldsymbol{A}_{\mu} \boldsymbol{A}^{\mu} \\
& =\frac{f_{\pi}^{2}}{2}\left[\left(\partial_{\mu} \boldsymbol{F} \cdot \partial^{\mu} \boldsymbol{F}\right) \frac{\sin ^{2} F}{F^{2}}+\left(\boldsymbol{F} \cdot \partial_{\mu} \boldsymbol{F}\right)\left(\boldsymbol{F} \cdot \partial^{\mu} \boldsymbol{F}\right) \frac{F^{2}-\sin ^{2} F}{F^{4}}\right],
\end{aligned}
$$

where we used

$$
\left[L_{\mu}, L_{\nu}\right]=-2 i \boldsymbol{\tau} \cdot\left(\boldsymbol{A}_{\mu} \times \boldsymbol{A}_{\nu}\right)
$$

and

$$
\operatorname{Tr}\left[L_{\mu}, L_{\nu}\right]^{2}=-8\left(\boldsymbol{A}_{\mu} \cdot \boldsymbol{A}^{\mu} \boldsymbol{A}_{\nu} \cdot \boldsymbol{A}^{\nu}-\boldsymbol{A}_{\mu} \cdot \boldsymbol{A}_{\nu} \boldsymbol{A}^{\mu} \cdot \boldsymbol{A}^{\nu}\right)
$$

for the Skyrme term

$$
\begin{aligned}
\mathcal{L}_{\mathrm{sk}}= & -\frac{1}{4 e^{2}}\left(\boldsymbol{A}_{\mu} \cdot \boldsymbol{A}^{\mu} \boldsymbol{A}_{\nu} \cdot \boldsymbol{A}^{\nu}-\boldsymbol{A}_{\mu} \cdot \boldsymbol{A}_{\nu} \boldsymbol{A}^{\mu} \cdot \boldsymbol{A}^{\nu}\right) \\
= & -\frac{1}{4 e^{2}}\left\{\frac{\sin ^{4} F}{F^{4}}\left[\left(\partial^{\mu} \boldsymbol{F} \cdot \partial_{\mu} \boldsymbol{F}\right)\left(\partial^{\nu} \boldsymbol{F} \cdot \partial_{\nu} \boldsymbol{F}\right)-\left(\partial^{\mu} \boldsymbol{F} \cdot \partial_{\nu} \boldsymbol{F}\right)\left(\partial^{\nu} \boldsymbol{F} \cdot \partial_{\mu} \boldsymbol{F}\right)\right]\right. \\
& +\frac{2 \sin ^{2} F\left(F^{2}-\sin ^{2} F\right)}{F^{6}}\left[\left(\partial^{\mu} \boldsymbol{F} \cdot \partial_{\mu} \boldsymbol{F}\right)\left(\boldsymbol{F} \cdot \partial_{\nu} \boldsymbol{F}\right)\left(\boldsymbol{F} \cdot \partial^{\nu} \boldsymbol{F}\right)\right. \\
& \left.\left.-\left(\partial^{\mu} \boldsymbol{F} \cdot \partial_{\nu} \boldsymbol{F}\right)\left(\boldsymbol{F} \cdot \partial_{\mu} \boldsymbol{F}\right)\left(\boldsymbol{F} \cdot \partial^{\nu} \boldsymbol{F}\right)\right]\right\},
\end{aligned}
$$

and for the pion mass term

$$
\mathcal{L}_{\mathrm{m}}=m_{\pi}^{2} f_{\pi}^{2}(\cos F-1)
$$

From the results above we can obtain the equation of motion from the usual starting point,

$$
\partial_{\mu} \frac{\partial \mathcal{L}}{\partial\left(\partial_{\mu} \boldsymbol{F}\right)}-\frac{\partial \mathcal{L}}{\partial \boldsymbol{F}}=0
$$

Since the equations of motion are long and complicated, the form in terms of $\boldsymbol{F}$ are displayed in the appendix.

In the next section we discuss the solution of these equations. Before turning to that section we record two other important formulae. The baryon number density is given by

$$
\mathcal{B}=-\frac{1}{2 \pi^{2}} \varepsilon_{\alpha \beta \gamma} \varepsilon^{i j k} A_{i}^{\alpha} A_{j}^{\beta} A_{k}^{\gamma} .
$$

Here $A$ is defined in Eq. for a system of Skyrmion and anti-Skyrmion. The energy density of the system is given by

$$
\mathcal{E}=\frac{f_{\pi}^{2}}{2} A_{i}^{\alpha} A_{i}^{\alpha}+\frac{1}{4 e^{2}}\left[\left(A_{i}^{\alpha} A_{i}^{\alpha}\right)^{2}-\left(A_{i}^{\alpha} A_{j}^{\alpha}\right)\left(A_{i}^{\beta} A_{j}^{\beta}\right)\right]+m_{\pi}^{2} f_{\pi}^{2}(1-\cos F)
$$

To find the total interaction energy of a Skyrmion and anti-Skyrmion configuration, we first solve the equations of motion for the static field configuration corresponding to our chosen constraints, (Skyrmion anti-Skyrmion separation and relative grooming) and then use that solved field configuration in the integrated energy density to obtain the interaction energy. Note the integrated energy is the total energy of the system. To obtain the interaction energy or the potentials, one must subtract the rest energy of the nucleon and antinucleon from that total energy. This is done by subtracting two large numbers to obtain what is usually a much smaller one, and hence requires considerable precision in the underlying calculation. Achieving that precision is the goal of the full Skyrme calculation and explains why it must be done so carefully. 


\section{Calculation}

Our aim is to obtain the minimized static energy of the Skyrmion and anti-Skyrmion separated by a certain distance and with a definite relative grooming. We need to consider the initial field configuration, the symmetry of the field, and constraints on it before embarking on solving the equations. The nonlinear nature of the equation of motion also brings the risk of numerical instabilities. These instabilities are more pronounced for Skyrmion-antiSkyrmion systems than for Skyrmion-Skyrmion due to the lack of a topological constraint in the Skyrmion-antiSkyrmion case that is due to annihilation.

The essence of our numerical method is to begin with a trial configuration that has the required symmetries, groomings and distance constraint. Then to use that configuration as the seed in the Skyrme equations of motion with a relaxation algorithm. The system will relax to the lowest energy Skyrme configuration compatible with the constraints. We begin with the symmetrized product ansatz for a groomed Skyrmion and a groomed anti-Skyrmion as initial configuration.

$$
U=\frac{1}{N}\left(U_{1} U_{2}+U_{2} U_{1}\right)
$$

where

$$
U_{1}=A \mathcal{U}\left(\vec{r}-\frac{1}{2} R \hat{x}\right) A^{\dagger}
$$

and

$$
U_{2}=A^{\dagger} \mathcal{U}^{\dagger}\left(\vec{r}+\frac{1}{2} R \hat{x}\right) A
$$

The $\mathrm{SU}(2)$ field $\mathcal{U}$ is that for a single Skyrmion in the defensive hedgehog configuration. The grooming matrix $A=\exp i(\theta / 4) \tau \cdot \hat{a}$ imparts a relative grooming of $\theta$ around direction $\hat{a}$ between the Skyrmion centered at $(R / 2) \hat{x}$ and the anti-Skyrmion at $-(R / 2) \hat{x}$. The normalization factor $N$ makes the symmetrized ansatz unitary.

Three essential groomings are needed to map the interaction energy of the Skyrmions into that of baryons. They are 1) no grooming; 2) relative grooming of $\pi$ around the $x$ axis (the axis separating the Skyrmion and anti-Skyrmion); and 3) relative grooming of $\pi$ around the $y$ or $z$ axis. Each of these groomings has symmetries that we exploit in order to simplify our calculation. The reflection symmetries (in the $\mathrm{x}, \mathrm{y}$ and $\mathrm{z}$ planes) of the fields are shown in Table 1 for the three situations.

These three grooming are the normal modes for the Skyrmion and anti-Skyrmion system and their symmetries should be maintained in solving the equation of motion. We achieve this by restricting our calculation to the first octant of the full three dimensional space, and use the reflection symmetries as boundary conditions for the field at the $\mathrm{x}, \mathrm{y}$ and $\mathrm{z}$ planes. This restriction also reduces our computational time by a factor of eight.

The anti-Skyrmion and Skyrmion system is not stable under the equation of motion unless it is subjected to a proper constraint. Since the total baryon number is zero for this system, the usual topological constraint used in the multi-Skyrmion case is not present here. For the Skyrmion-Skyrmion, the distance $R$ between the two, as defined in [9] by

$$
\frac{1}{4} R^{2}=\int d^{3} r \mathcal{B}(\vec{r}) r^{2}
$$

is constrained to a definite value. For the Skyrmion-antiSkyrmion, this can no longer be used. The baryon density is odd across the $x$-plane and hence the distance $R$ obtained from such a density is obviously zero. The constraint we propose instead is a topological one. When the separation (as defined in the symmetrized product ansatz) is more than $0.8 \mathrm{fm}$, the absolute value of the chiral angle always has a peak value of $\pi$ at two places on the $\mathrm{x}$-axis for all three of the groomings. Note that these locations are not $\pm(R / 2) \hat{x}$, although they are asymptotically for very large separation. At these two locations, we hold the field values constant as defined in the symmetrized product ansatz when we solve the equation of motion. We use the separation value of $R$ as defined in the symmetrized product ansatz. For distance less than $0.8 \mathrm{fm}$, the solution becomes numerically less tractable and a definition of separation is unclear. We do not study interaction energy of these cases in the 


\begin{tabular}{|c|c|c|c|}
\hline & $\mathrm{x}$ & $\mathrm{y}$ & $\mathrm{z}$ \\
\hline \hline \multicolumn{5}{|c|}{ grooming } \\
\hline$F_{1}$ & $\mathrm{E}$ & $\mathrm{E}$ & $\mathrm{E}$ \\
\hline$F_{2}$ & $\mathrm{O}$ & $\mathrm{O}$ & $\mathrm{E}$ \\
\hline$F_{3}$ & $\mathrm{O}$ & $\mathrm{E}$ & $\mathrm{O}$ \\
\hline \hline \multicolumn{5}{|c}{$\mathrm{x}-\pi$} \\
\hline$F_{1}$ & $\mathrm{E}$ & $\mathrm{E}$ & $\mathrm{E}$ \\
\hline$F_{2}$ & $\mathrm{E}$ & $\mathrm{E}$ & $\mathrm{O}$ \\
\hline$F_{3}$ & $\mathrm{E}$ & $\mathrm{O}$ & $\mathrm{E}$ \\
\hline \hline \multicolumn{5}{|c}{$\mathrm{z}-\pi$} \\
\hline$F_{1}$ & $\mathrm{E}$ & $\mathrm{O}$ & $\mathrm{E}$ \\
\hline$F_{2}$ & $\mathrm{O}$ & $\mathrm{E}$ & $\mathrm{E}$ \\
\hline$F_{3}$ & $\mathrm{O}$ & $\mathrm{E}$ & $\mathrm{O}$ \\
\hline
\end{tabular}

Table 1: Symmetry of the three groomed configurations. The E and O label the even or odd symmetry of the particular field component across the $\mathrm{x}, \mathrm{y}$ or $\mathrm{z}$ plane.

present paper. Such small separations are not meaningful for the nucleon antinucleon problem since empirically one finds complete annihilation well before that distance is reached. As we shall see below, we also find very strong coupling to annihilation below $1 \mathrm{fm}$.

Using the symmetry and topological constraints discussed above, we solve the equations of motion in the first octant of coordinates to obtain the minimized energy. To do so, we reduce the equation of motion to a dissipative one by setting all first order time derivatives to zero at each time step. The resulting Langevin type equation is solved on a lattice in the first octant with appropriate boundary conditions on the faces that form the boundaries of the octant. We propagate the field in discrete time steps until a stable final field configuration is reached. The field profile is strongly peaked around the locations where the chiral angle reaches the value of $\pi$. We use a lattice coordinate system with variable grid that has the densest distribution of points at these locations.

\section{Results}

In this section we present the results of our numerical calculations of the Skyrmion-antiSkyrmion interaction and of the corresponding nucleon-antinucleon interaction. We begin with the interaction energy of the SkyrmionantiSkyrmion $(S \bar{S})$ system in the three groomings required to extract the nucleonic potentials, namely the $S \bar{S}$ system with no relative grooming, the system with a relative grooming of $\pi$ around the axis joining the $S$ and $\bar{S}$ (the x-axis) and the system with a relative grooming of $\pi$ along an axis at right angles to the line connecting the $S$ and $\bar{S}$ (z-axis). The results of our calculation are shown in Figure 1 . The Figure shows the total energy of the $S \bar{S}$ system as a function of separation distance. Note that for large separation the total energy is that of two free Skyrmions, namely $2.92 \mathrm{GeV}$ for our choice of parameters. The separation distance is defined as discussed in Section 3. It includes a scheme for holding the pion field at its maximum value at particular points. We only show a portion of the separation distance in the figure since for distances larger than $2.2 \mathrm{fm}$, the interaction is nearly zero and for distances below $0.8 \mathrm{fm}$, our entire scheme as well as the meaning of adiabatic interaction ceases to make sense. It is important to note that the "holding" process is essential to 
our calculation, since without it all $S \bar{S}$ configurations would relax to the lowest total energy of such a system, namely zero corresponding to total annihilation. In this sense our calculation is very different from that of Walhout and Wambach for the $S-S$ system [9].

From Figure 1 we see that the x-grooming channel (grooming along the axis joining the two) is attractive while grooming along the orthogonal axis is repulsive. This is just the opposite of what happens for the $S-S$ system, as we might expect from g-parity or related arguments. The no grooming channel is also repulsive. Well below $0.8 \mathrm{fm}$ it must turn attractive again since for zero separation and no grooming there is complete annihilation and the total energy must be zero. The results in Figure 1 should be compared with our previous results calculated with the product ansatz [7]. The qualitative results are the same. In particular the results agree at large distances where we expect the product ansatz to be a good approximation. However the full calculation with dynamical relaxation of the fields has much more attraction in the attractive channel and less repulsion in the repulsive channel as compared with the product ansatz. All this suggests, correctly as we will see below, that the full calculation will yield the strong $N-\bar{N}$ midrange attraction seen phenomenologically, but missing in the product ansatz.

The full dynamical results shown in Figure 1 suggest that for the $S \bar{S}$ system with no grooming there is a point of unstable equilibrium inside $0.8 \mathrm{fm}$. If the $S \bar{S}$ system is released from rest just inside that unstable point, it will proceed to annihilate, while if it is released just outside, it will separate to infinity. Such a critical radius should continue to exist at scattering energies such that at impact parameters less than a certain value annihilation occurs while for impact parameters greater than that value, the $S$ and $\bar{S}$ emerge at infinity. It should be emphasized that this is a classical scattering problem, so that no probabilities are allowed and that, due to the topology, the Skyrmion must appear at infinity in its entirety or not at all. The existence of such a critical impact parameter seems not to have been noted before, and reveals the existence of a new singular length in the Skyrme model. Scattering exactly at the singular impact parameter must involve very long time delays.

Some sense of the significance of the three groomings can be gathered by looking at arrow plots. These are plots of the chiral angle field, $\boldsymbol{F}$, at various points in a plane through the $S$ and $\bar{S}$. We use the x-y plane. Recall that $F \rightarrow 0$ at large distances and at all places where the energy density is small. We show the arrow plots for the three groomings, always with a $S \bar{S}$ separation of $1 \mathrm{fm}$. in Figure 2. Figure 2a shows the no grooming case. The $S$ hedgehog is on the left and the $\bar{S}$ anti-hedgehog on the right. The attractive channel, with grooming along the x-axis that joins the $S$ and $\bar{S}$ is shown in Figure 2b. It is clear that most of the $\boldsymbol{F}$ arrows are now quite short, corresponding to reduced energy density and thus attraction. Finally the repulsive channel with grooming along the z-axis is shown in Figure 2c. Here not only do the arrows not get short, but it is clear that as the $S$ and $\bar{S}$ approach the opposite arrows will clash leading to repulsive energy at short distances.

We now turn to extracting the nucleon antinucleon interaction from the $S \bar{S}$ results. As we have emphasized before, [7, 10], there are two steps in this process. First from combinations of the various $S \bar{S}$ groomings we can construct matrix elements of the baryon anti-baryon interaction. These include not just the nucleon-antinucleon matrix elements but also matrix elements coupling to the $\Delta$ resonance and diagonal matrix elements involving the $\Delta$ 's. The observed nucleon-antinucleon interaction involves these coupled terms since all that we are certain of experimentally is that there are nucleons and anti-nucleons asymptotically. When they get close and begin to interact, $\Delta$ 's are allowed to mix in. The formalism for including that mixing using the Born-Oppenheimer approximation has been presented in detail before, [7, 10], and we do not repeat it here. Rather we just give the results for the various nucleon antinucleon interactions showing both the results from taking only the nucleonic projections and the results from including the full mixing. We will see, as we expect, that the diagonalized Born-Oppenheimer mixing leads to enhanced attraction. We also include some finite $N_{C}$ corrections for $N_{C}=3$ in our calculation, as we did before. 
Figure 3 shows the nucleon antinucleon central interaction in the $T=0$ channel. We show the result from taking only the nucleon projection as well as the result from the full Born-Oppenheimer diagonalization. Both show much more attraction than we found in the case of the product ansatz, with the diagonalization significantly increasing the attraction. With diagonalization, the potential is about as strong as that seen phenomenologically either in the Bryan-Phillips analysis, [1], or in that of the Nijmegen group, [12], but the dependence on $R$ is somewhat different. In all cases, the potential is very strong. Note that the potentials do not mean much inside of $1 \mathrm{fm}$ since there is very strong absorption there due to annihilation. Figure 4 shows the same results for the central but $T=1$ channel. Due to the much greater effect of $\Delta$ mixing in this channel, the effect of diagonalization is to produce much more attraction. Now the full calculated potential is comparable to or perhaps even more attractive than the phenomenological results, but most of the overshoot is at the short distances where the potential concept is suspect. Again we find the correct scale (about half of the scale of the $T=0$ case) of the central midrange attraction and again far more attraction than we found in the product ansatz case. The spin dependent potentials are shown in Figures 5 and 6 . For the $T=0$ case the agreement with the phenomenological results is satisfactory. The product ansatz calculation gave the completely wrong answer for this case. For the $T=1$ spin dependent potential, the results are not as good, but the smallness of the potential is reproduced. In our calculation, that small value arises from the cancelation of a number of large factors and hence is very sensitive to details. The $T=0$ tensor force (Figure 7 ) is in nearly perfect agreement with the phenomenology and the $T=1$ tensor force (Figure 8) is rather well given, particularly at the larger distances. The tensor force is dominated by one pion exchange and hence was also well accounted for in the product ansatz.

Thus far we have concentrated on the nucleon antinucleon interaction potentials. But a defining feature of the baryon anti-baryon system is annihilation. Phenomenologically this can be represented in the initial state by an optical or absorptive potential. The data suggests that that potential is very strong, but short ranged. That is it sets in sharply at around $1 \mathrm{fm} 13$. In the classical Skyrme picture we would expect to see this as a sudden drop in the local baryon number. That is, consider the baryon number in the half space where the Skyrmion sits. Asymptotically that number should be one. Due to the finite size of our lattice, we would find a number slightly less than one. In Figure 9 we plot that baryon number as a function of Skyrmion anti-Skyrmion separation for the three groomings. We see that it is indeed very near one for large separations. In the repulsive channel it remains near one even for relatively small separations $(1 \mathrm{fm})$. In the attractive channel we see something very different. The baryon number begins near one at large separation, but near 1.2 $\mathrm{fm}$ it plunges abruptly to a value very near zero. This is annihilation. The fact that it occurs so sharply and at roughly the distance required by the data give further support for the Skyrme picture. In the channel with no grooming there is an even sharper onset of annihilation, but at a somewhat smaller distance. In our subsequent work we will use this annihilation mechanism combined with a coupled channel quantum approach using the interaction potentials we have obtained here to model the initial state.

All in all the nucleon antinucleon interaction extracted from the full, dynamical Skyrmion anti-Skyrmion interaction agrees well with the major trends of the data, and in particular reproduces the strong central attraction seen in the region between 1 and $2 \mathrm{fm}$, and the rapid onset of annihilation. This demonstrates that the Skyrme approach can account for the nucleon antinucleon initial state, as well as the final annihilation state.

\section{Discussion}

We have shown that the strong central attraction, the sharp onset of annihilation at about $1 \mathrm{fm}$ and the other the principal features of the phenomenological nucleon antinucleon interaction emerge from a careful calculation 
of that interaction based on the Skyrme model. Since the Skyrme picture models QCD in the classical or large number of colors and low energy limit and since that limit is the appropriate one for low energy nonperturbative phenomena, our calculation links low energy nucleon antinucleon interactions to QCD. There are two essential pieces to our calculation. The first is a careful and thorough dynamical (though static) computation of the Skyrmion anti-Skyrmion configurations. It is this step that is vital to getting the strong, midrange attraction missing from the product ansatz approach to the same problem. This dynamical calculation is complex and difficult and represents the major new work presented here. The second step involves using not just Skyrmion to nucleon projections but also state mixing to obtain the full nucleon interaction. We have used these two parts to obtain a very satisfactory account of the nucleon anti-nucleon interaction.

Previously we have shown that the Skyrme model can account for the major features of the annihilation branches in low energy nucleon antinucleon annihilation, [5], but that calculation had no initial state dynamics. In this paper we have shown that the initial state can also be successfully studied using the Skyrme approach. Our next step is to combine these two and give a complete, QCD based (via Skyrme) description of low energy nucleon antinucleon annihilation from start to finish. We plan to turn to that task next.

\section{Acknowledgement}

The authors wish to thank Dr. Niels R. Walet for his interest in this project and in particular for his help in emphasizing the importance of symmetries in simplifying the calculation. This work was supported in part by a grant from the National Science Foundation.

\section{References}

[1] T.H.R. Skyrme, Proc. R. Soc. London 262, 237 (1961); Nucl. Phys. 31, 556 (1962).

[2] G. 't Hooft, Nucl. Phys. B 72, 461 (1974); B 75, 461 (1974).

[3] E. Witten, Nucl. Phys. B 160, 57 (1979), ibid B 223, 433 (1983).

[4] H.M. Sommermann, R. Seki, S. Larson and S.E. Koonin, Phys. Rev. D 45, 4303 (1992).

[5] Yang Lu and R.D. Amado, Phys. Lett. B 357, 446 (1995); Phys. Rev. C 52, 2158 (1995).

[6] W. Y. Crutchfield and J. B. Bell, J. Comp. Phys. 110, 234 (1994).

[7] Yang Lu and R.D. Amado, Phys. Rev. C 54, 1566 (1996).

[8] R. Vinh Mau, M. Lacombe, B. Loiseau, W. N. Cottingham, and P. Lisboa, Phys. Lett. 150B, 259 (1985).

[9] T.S. Walhout and J. Wambach, Phys. Rev. Lett. 67, 314 (1991).

[10] N. R. Walet and R. D. Amado, Phys. Rev. C 47, 498 (1993).

[11] R.A. Brian and R.J.N. Phillips, Nucl. Phys. B5, 201 (1968).

[12] M.M. Nagels et al., Phys. Rev. D 12, 744 (1975); P.H. Timmers et al., Phys. Rev. D 29, 1928 (1984).

[13] C. Amsler and F. Myhrer, Annual Rev. Nucl. Part. Sci. 41, 219 (1991) 


\section{A Equation of motion}

The equation of motion in the chiral angle $\boldsymbol{F}$ is a long expression. It contain three terms. The term from the nonlinear sigma model is

$$
\begin{aligned}
f_{\pi}^{2} & {\left[\partial_{\mu} \partial^{\mu} \boldsymbol{F} \frac{\sin ^{2} F}{F^{2}}\right.} \\
+ & \boldsymbol{F}\left(\boldsymbol{F} \cdot \partial_{\mu} \partial^{\mu} \boldsymbol{F}\right) \frac{F^{2}-\sin ^{2} F}{F^{4}} \\
+ & \boldsymbol{F}\left(\partial^{\mu} \boldsymbol{F} \cdot \partial_{\mu} \boldsymbol{F}\right) \frac{F-\sin F \cos F}{F^{3}} \\
+ & 2 \partial_{\mu} \boldsymbol{F}\left(\boldsymbol{F} \cdot \partial^{\mu} \boldsymbol{F}\right) \frac{\sin F(F \cos F-\sin F)}{F^{4}} \\
+ & \left.\boldsymbol{F}\left(\boldsymbol{F} \cdot \partial^{\mu} \boldsymbol{F}\right)\left(\boldsymbol{F} \cdot \partial_{\mu} \boldsymbol{F}\right) \frac{2 \sin ^{2} F-F^{2}-F \sin F \cos F}{F^{6}}\right] .
\end{aligned}
$$

The term from the Skyrme term is

$$
\begin{aligned}
& -\frac{1}{e^{2}}\left\{\quad \frac{\sin ^{4} F}{F^{4}}\left[\partial_{\mu} \partial^{\mu} \boldsymbol{F}\left(\partial^{\nu} \boldsymbol{F} \cdot \partial_{\nu} \boldsymbol{F}\right)-\partial_{\mu} \partial^{\nu} \boldsymbol{F}\left(\partial^{\mu} \boldsymbol{F} \cdot \partial_{\nu} \boldsymbol{F}\right)\right]\right. \\
& +\frac{\sin ^{2} F\left(F^{2}-\sin ^{2} F\right)}{F^{6}}\left[\partial_{\mu} \partial^{\mu} \boldsymbol{F}\left(\boldsymbol{F} \cdot \partial_{\nu} \boldsymbol{F}\right)\left(\boldsymbol{F} \cdot \partial^{\nu} \boldsymbol{F}\right)-\partial_{\mu} \partial^{\nu} \boldsymbol{F}\left(\boldsymbol{F} \cdot \partial^{\mu} \boldsymbol{F}\right)\left(\boldsymbol{F} \cdot \partial_{\nu} \boldsymbol{F}\right)\right. \\
& +\partial^{\mu} \boldsymbol{F}\left(\left(\boldsymbol{F} \cdot \partial_{\mu} \partial_{\nu} \boldsymbol{F} \partial^{\nu} \boldsymbol{F}-\left(\boldsymbol{F} \cdot \partial^{\nu} \partial_{\nu} \boldsymbol{F}\right)\left(\left(\boldsymbol{F} \cdot \partial_{\mu} \boldsymbol{F}\right)\right)\right.\right. \\
& +\boldsymbol{F}\left(\left(\boldsymbol{F} \cdot \partial^{\mu} \partial_{\mu} \boldsymbol{F}\right)\left(\partial^{\nu} \boldsymbol{F} \cdot \partial_{\nu} \boldsymbol{F}\right)-\left(\boldsymbol{F} \cdot \partial^{\mu} \partial_{\nu} \boldsymbol{F}\right)\left(\boldsymbol{F} \cdot \partial_{\mu} \partial^{\nu} \boldsymbol{F}\right)\right) \\
& \left.+\boldsymbol{F}\left(\left(\boldsymbol{F} \cdot \partial^{\mu} \boldsymbol{F}\right)\left(\partial^{\nu} \boldsymbol{F} \cdot \partial_{\mu} \partial_{\nu} \boldsymbol{F}\right)-\left(\boldsymbol{F} \cdot \partial^{\mu} \boldsymbol{F}\right)\left(\partial_{\mu} \boldsymbol{F} \cdot \partial_{\nu} \partial^{\nu} \boldsymbol{F}\right)\right)\right] \\
& +\left[\frac{\sin ^{2} F}{F^{4}}-\frac{\sin ^{3} F \cos F}{F^{5}}\right] \boldsymbol{F}\left[\left(\partial^{\mu} \boldsymbol{F} \cdot \partial_{\mu} \boldsymbol{F}\right)\left(\partial^{\nu} \boldsymbol{F} \cdot \partial_{\nu} \boldsymbol{F}\right)-\left(\partial^{\mu} \boldsymbol{F} \cdot \partial_{\nu} \boldsymbol{F}\right)\left(\partial^{\nu} \boldsymbol{F} \cdot \partial_{\mu} \boldsymbol{F}\right)\right] \\
& +\left[4 \frac{\sin ^{3} F \cos F}{F^{5}}-3 \frac{\sin ^{4} F}{F^{6}}-\frac{\sin ^{2} F}{F^{4}}\right] \\
& \partial^{\mu} \boldsymbol{F}\left[\left(\boldsymbol{F} \cdot \partial_{\mu} \boldsymbol{F}\right)\left(\partial_{\nu} \boldsymbol{F} \cdot \partial^{\nu} \boldsymbol{F}\right)-\left(\boldsymbol{F} \cdot \partial_{\nu} \boldsymbol{F}\right)\left(\partial_{\mu} \boldsymbol{F} \cdot \partial^{\nu} \boldsymbol{F}\right)\right] \\
& +\left[\frac{\sin F \cos F}{F^{5}}-2 \frac{\sin ^{3} F \cos F}{F^{7}}-2 \frac{\sin ^{2} F}{F^{6}}+3 \frac{\sin ^{4} F}{F^{8}}\right] \\
& \left.\boldsymbol{F}\left[\left(\partial_{\mu} \boldsymbol{F} \cdot \partial^{\mu} \boldsymbol{F}\right)\left(\boldsymbol{F} \cdot \partial_{\nu} \boldsymbol{F}\right)\left(\boldsymbol{F} \cdot \partial^{\nu} \boldsymbol{F}\right)-\left(\partial_{\mu} \boldsymbol{F} \cdot \partial^{\nu} \boldsymbol{F}\right)\left(\boldsymbol{F} \cdot \partial_{\nu} \boldsymbol{F}\right)\left(\boldsymbol{F} \cdot \partial^{\mu} \boldsymbol{F}\right)\right]\right\},
\end{aligned}
$$

and finally the term with the pion mass is

$$
-m_{\pi}^{2} f_{\pi}^{2} \frac{\sin F}{F} \boldsymbol{F} .
$$

The equation of motion is given by equating the sum of these three terms to zero. 


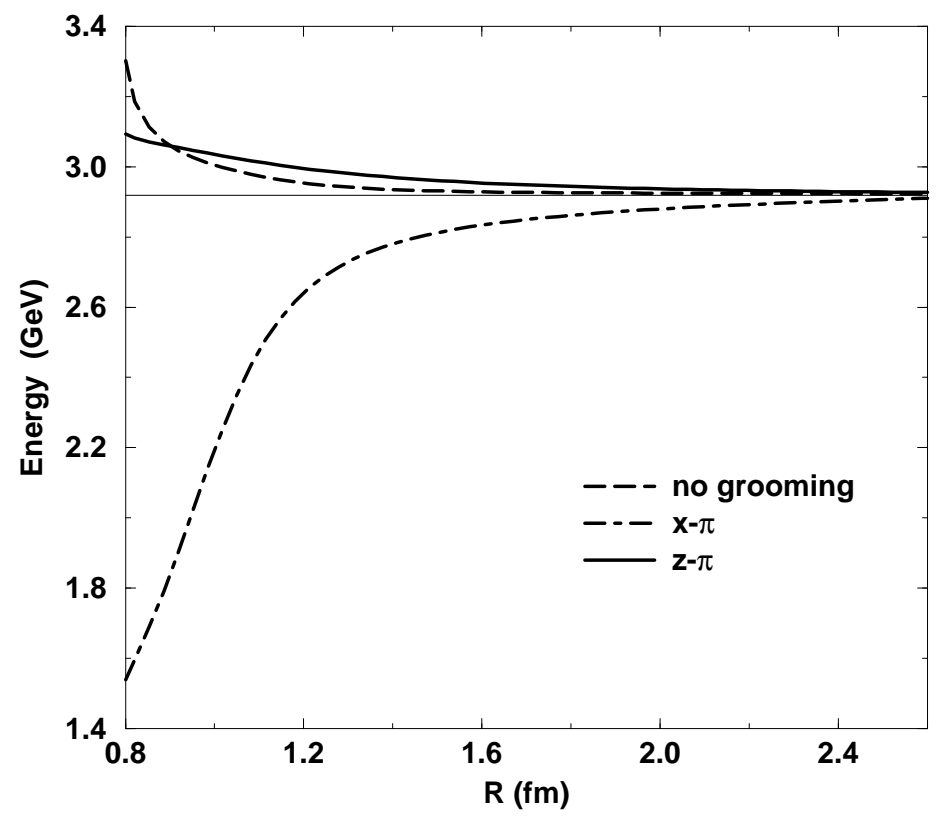

Figure 1: Total energy of the $S \bar{S}$ system as a function of separation distance for three major groomings. The horizontal line represents twice the Skyrmion mass, at $2.92 \mathrm{GeV}$. 


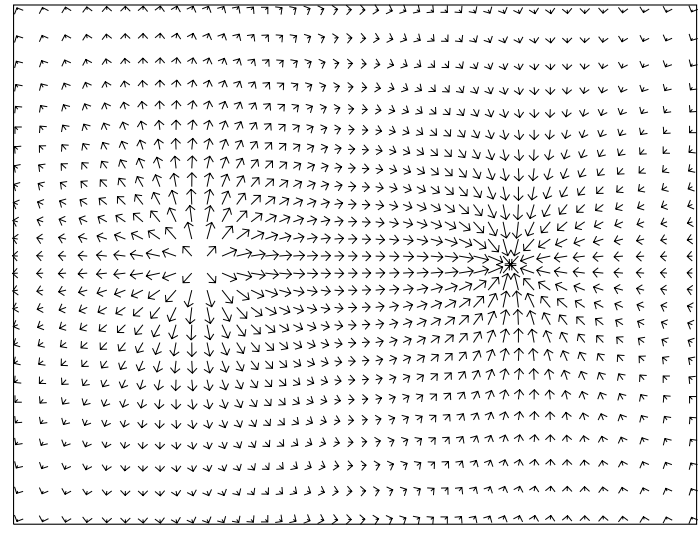

$2 \mathrm{a}$

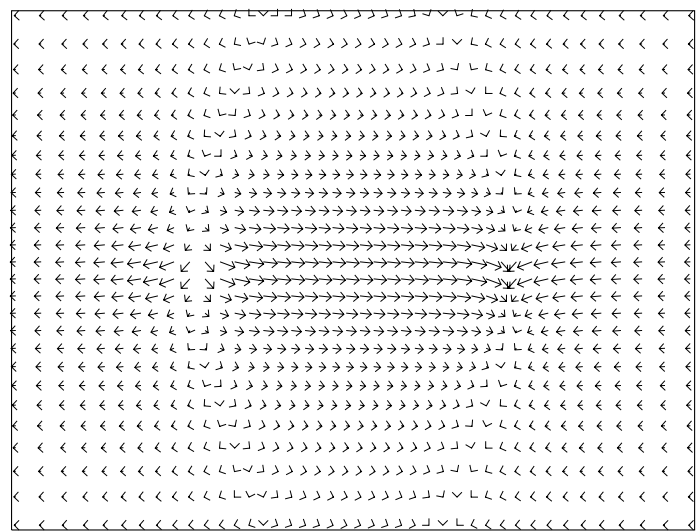

$2 \mathrm{~b}$

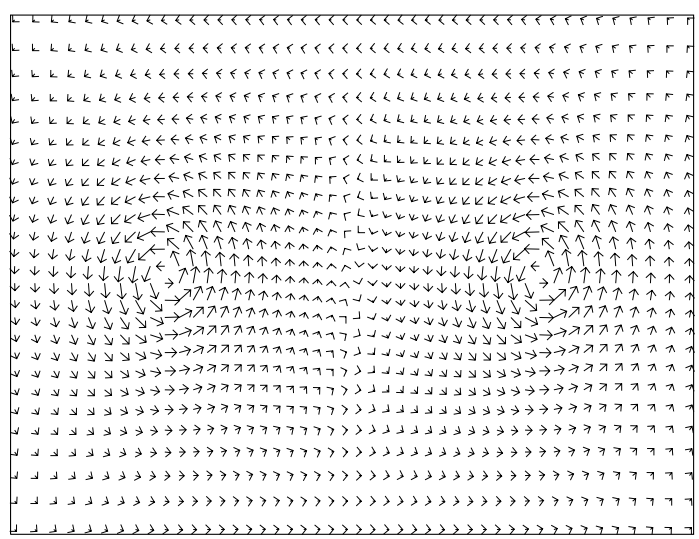

$2 \mathrm{c}$

Figure 2: Arrow plots of the chiral angle field for the three groomings at separation of $1 \mathrm{fm}$. Figure 2a shows the no grooming case, $2 \mathrm{~b}$ for grooming of $\pi$ around $\mathrm{x}$-axis and $2 \mathrm{c}$ grooming of $\pi$ around z-axis. The Skyrmion is on the left and the anti-Skyrmion on the right. 


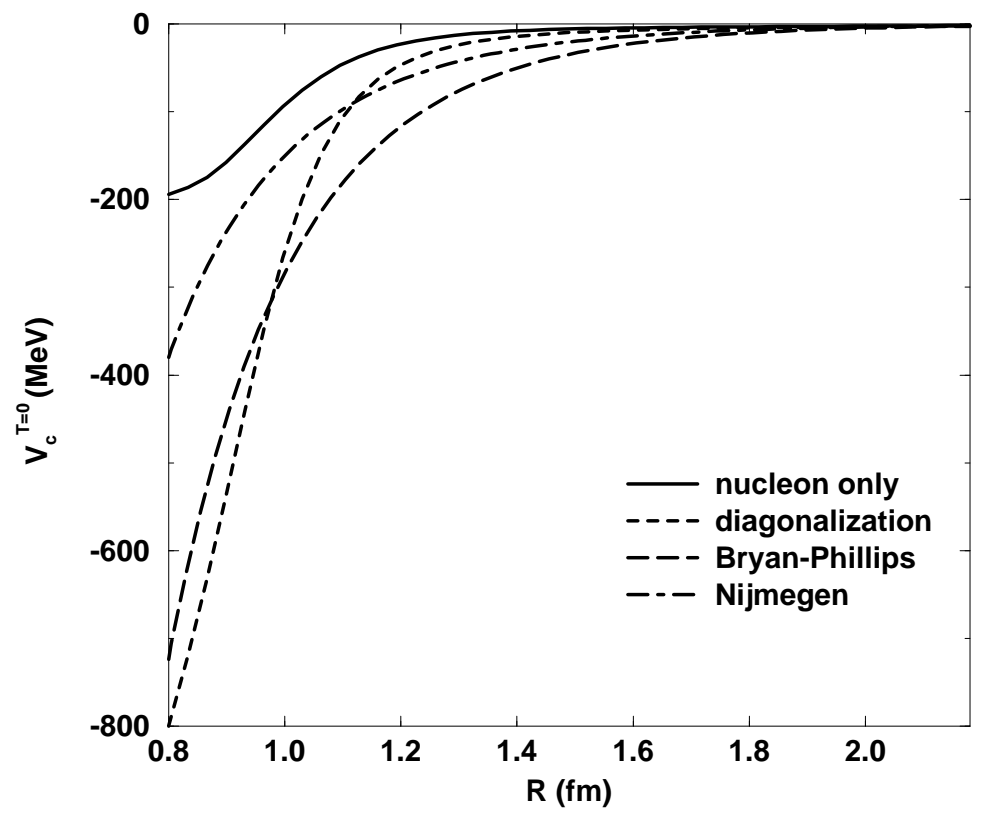

Figure 3: Central potential $V_{C}^{T}$ as a function of $R$ in the region $0.8-2.2 \mathrm{fm}$ for the $T=0$ channels. The solid line gives the nucleons only result from minimization. The short dashed line is the result of the state mixing using full Born-Oppenheimer diagonalization. The phenomenological potentials based on meson exchange are shown by the long-dashed line for Bryan-Phillips potential [1] and by the dash-dotted line for the Nijmegen potential [12]. 


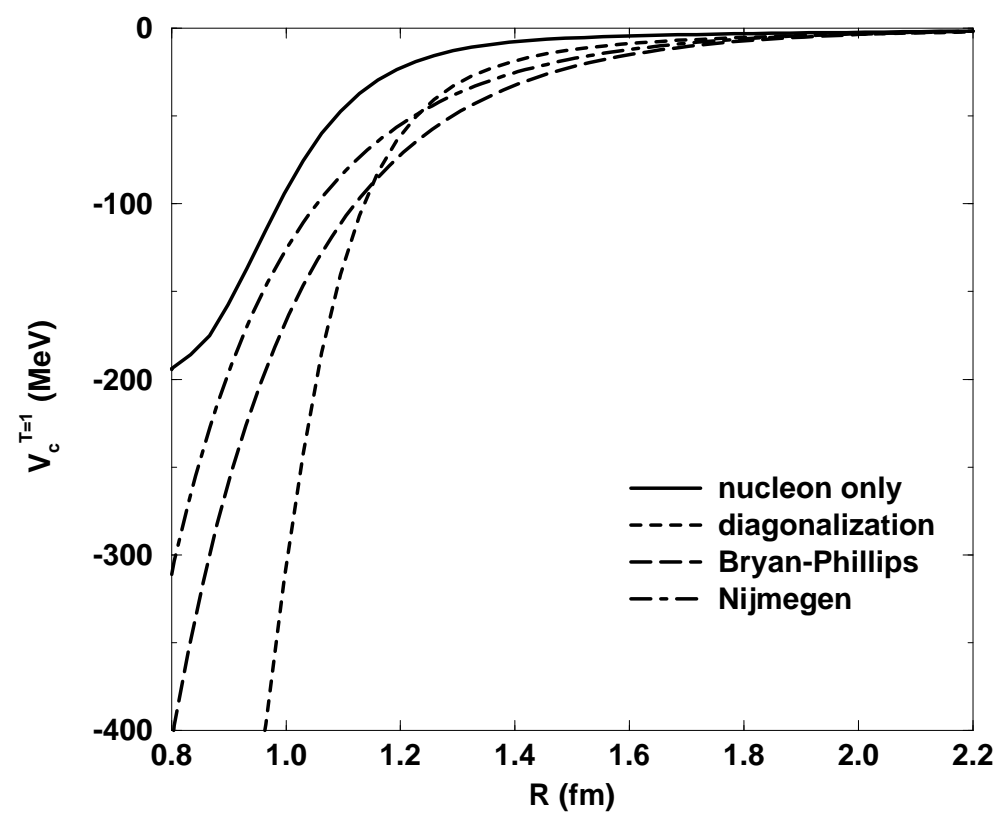

Figure 4: Central potential, same as in Fig. 3 but for $T=1$.

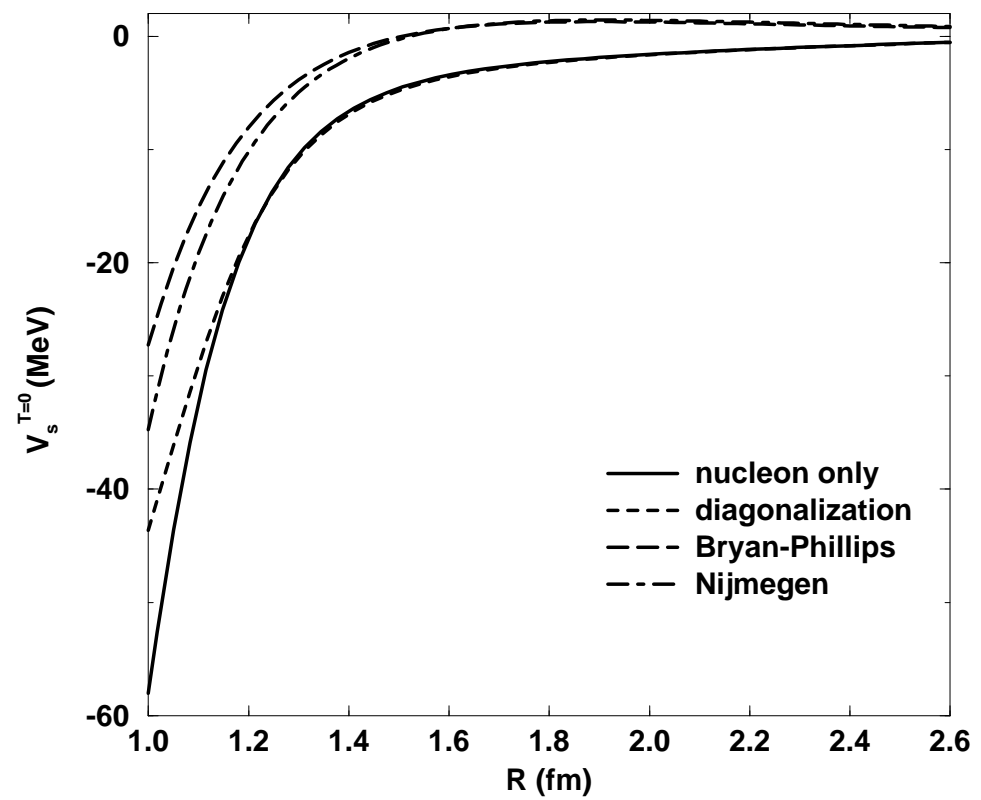

Figure 5: The spin dependent potential $V_{s}$ as a function of $R$ in the region 1-2.6 $\mathrm{fm}$ for $T=0$. Labeling of curves is the same as in Fig. 3. 


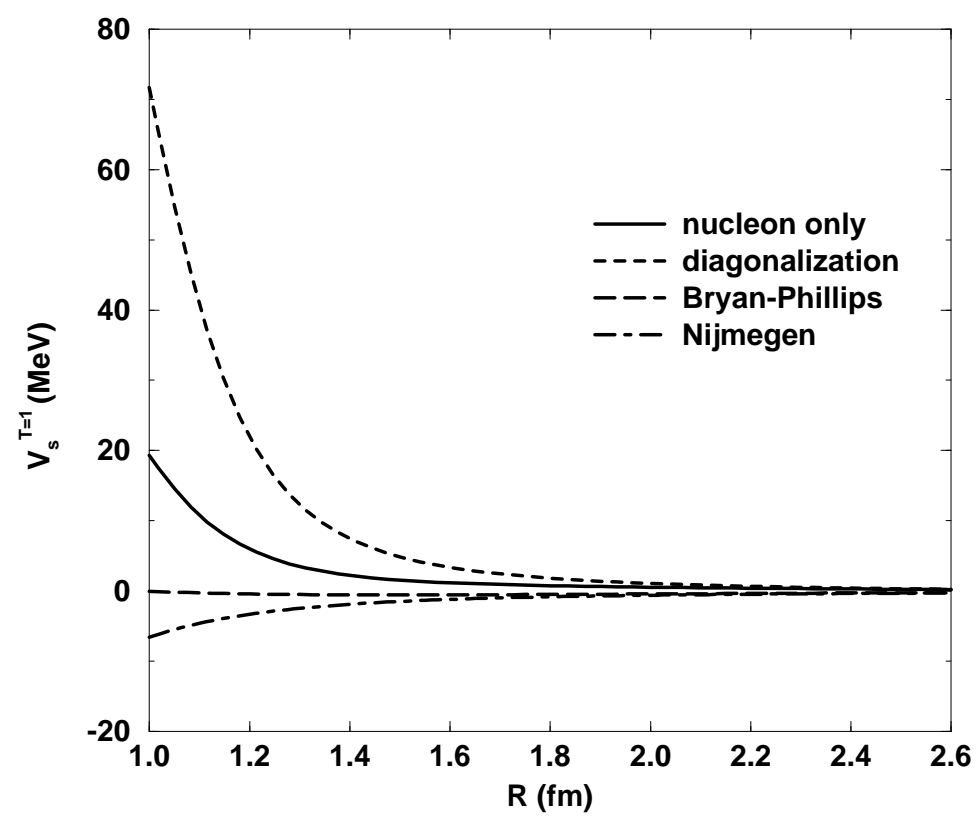

Figure 6: Spin-dependent potential, same as Fig. 5 but for $T=1$.

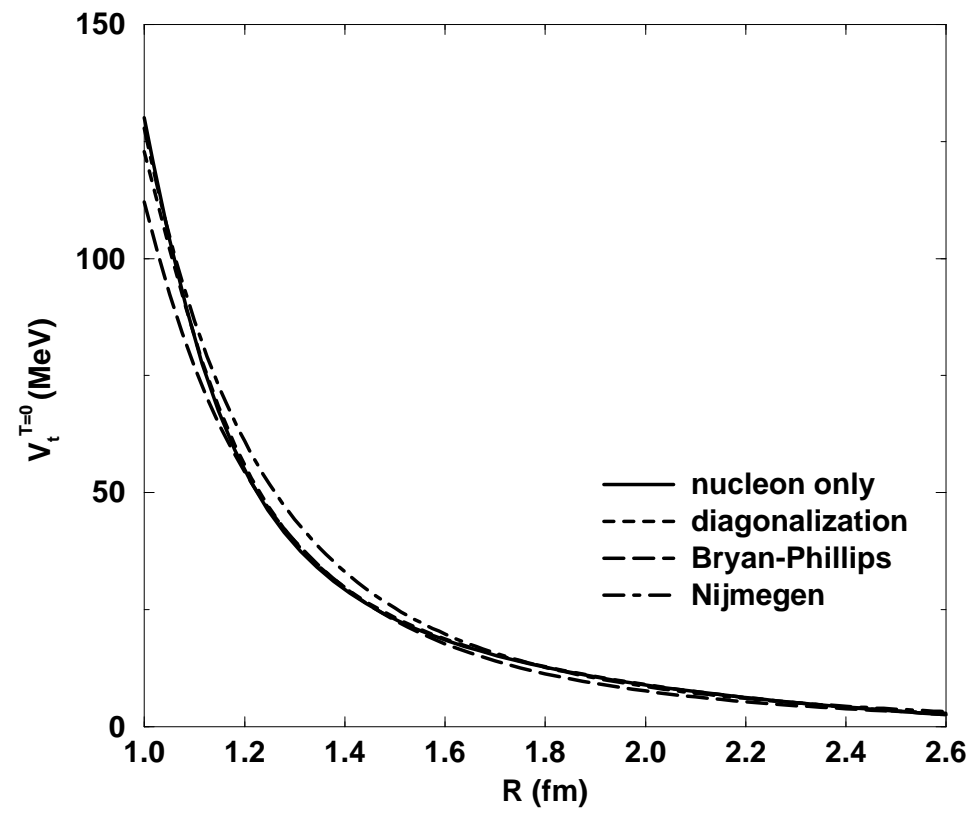

Figure 7: Tensor potential $V_{t}$ as a function of $R$ in the region 1-2.6 fm for $T=0$. Labeling of curves is the same as in Fig. 3. 


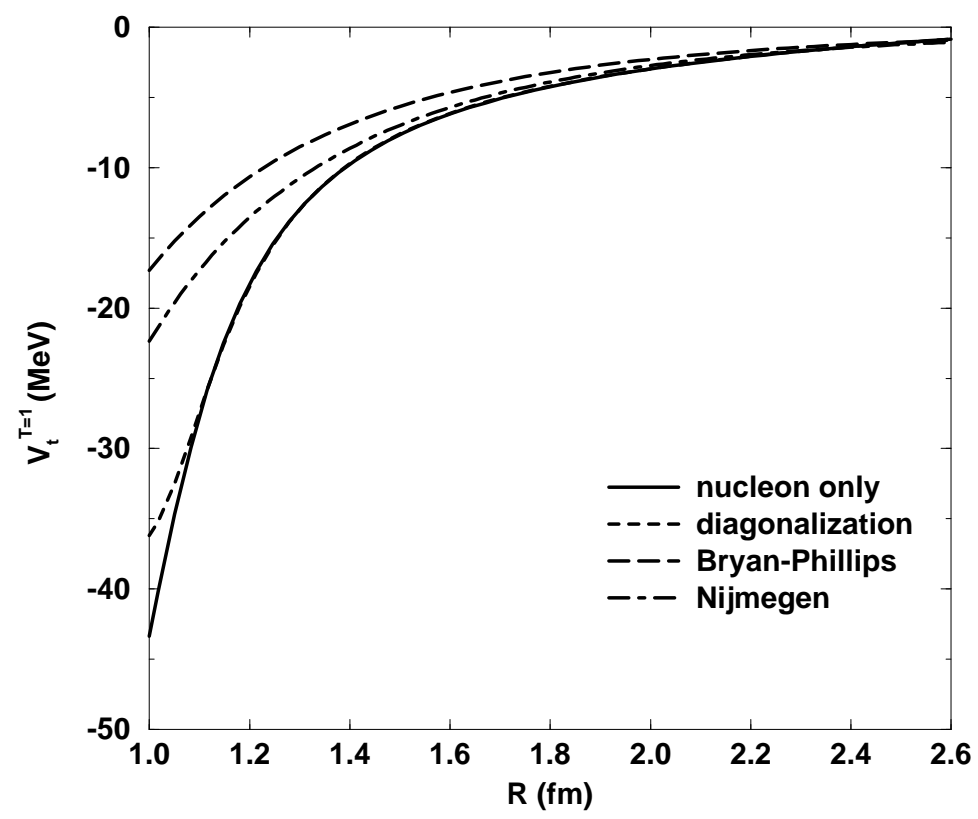

Figure 8: Tensor potential, same as in Fig. 7 but for $T=1$.

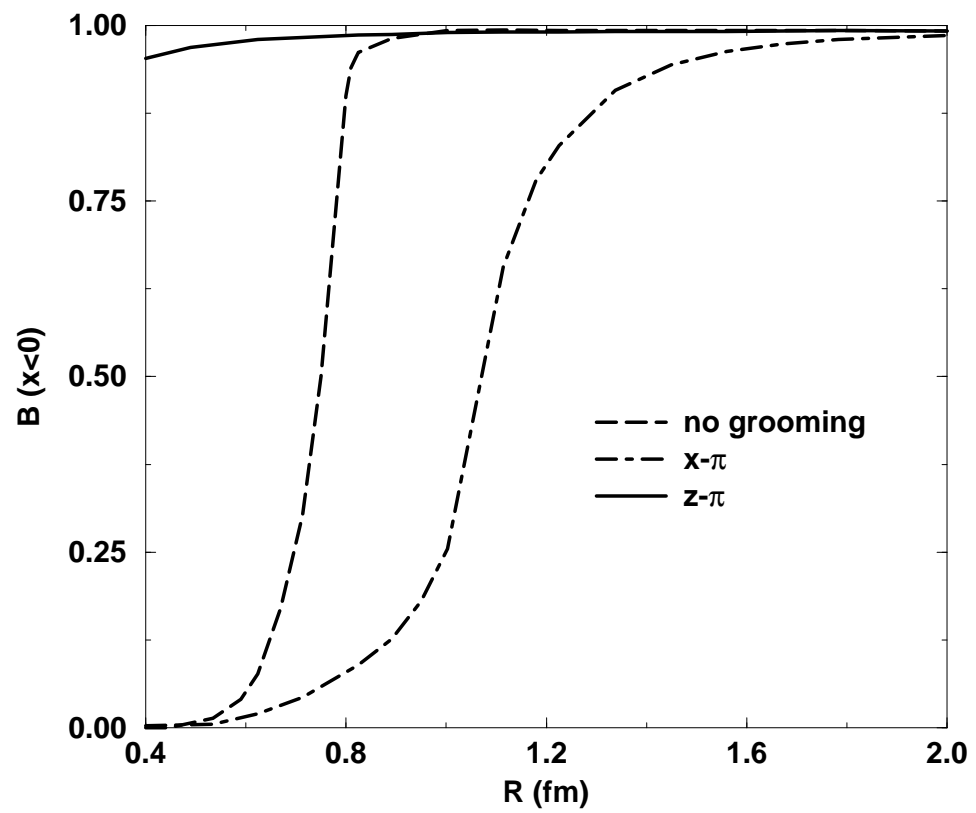

Figure 9: Baryon number in the half of space $(x<0)$ where the Skyrmion resides, for three major groomings, as a function of separation. 


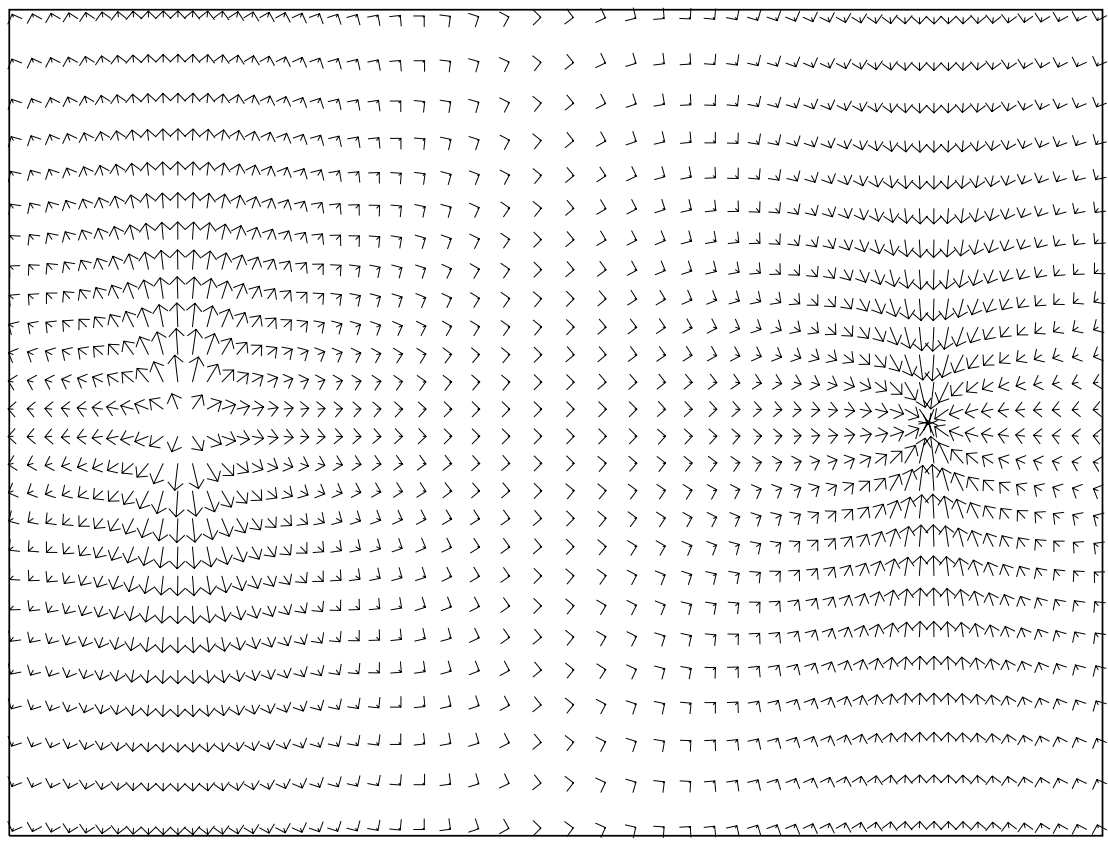

